EXTENDED REPORT

\title{
Is avoidant coping independent of disease status and stable over time in patients with ankylosing spondylitis?
}

\author{
A Boonen, D van der Heijde, R Landewé, A Chorus, W van Lankveld, H Miedema, \\ $\mathrm{H}$ van der Tempel, $\mathbf{S}$ van der Linden
}

Ann Rheum Dis 2004;63:1264-1268. doi: 10.1136/ard.2003.012435

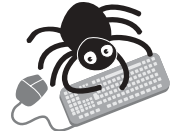

The appendices can be found on the journal website (http:// www.annrheumdis.com/ supplemental)

See end of article for authors' affiliations

\section{Correspondence to:} Dr A Boonen, Department of Internal Medicine, Division of Rheumatology, University Hospital Maastricht, PO Box 5800, 6202 AZ Maastricht, Netherlands; aboo@sint.azm.n

Accepted 1 December 2003
Objective: To determine whether avoidant coping in ankylosing spondylitis (AS) is independent of disease status and whether it is stable over time.

Methods: 658 patients with AS completed a postal questionnaire on health status, including pain and stiffness (BASDAl), physical function (BASFI), and coping (CORS). In CORS, "decreasing activities to cope with pain" and "pacing to cope with limitations" reflect avoidant behavioural coping. Ninety patients continued in a longitudinal study and 70 completed the CORS after four years. The adjusted contribution of age, sex, disease duration, educational level, pain (BASDAl), and physical function (BASFI) to the two avoidant coping strategies at first assessment was determined by multiple linear regression. Agreement between coping at first assessment and four years later was determined by intraclass correlation, and the correlation between change in coping and change in disease status over time by Pearson's correlation. Results: At first assessment, worse physical function (BASFI) and more pain (BASDAl) were associated with "decreasing activities to cope with pain". Worse physical function, but not pain, was associated with "pacing to cope with limitations". The contribution of physical function or pain to the total explained variance in each of the coping strategies was small. Disease duration was not a determinant of avoidant coping, but greater age was associated with "pacing to cope with limitations". Change in avoidant coping strategies over time could not be explained by change in function or pain.

Conclusions: In AS, avoidant coping at a particular time is largely independent of disease duration or status. Variability in avoidant coping over a limited period of four years cannot be explained by change in disease status.
C oping is a cognitive or behavioural effort to manage specific external or internal demands. ${ }^{12}$ Several models have been proposed to elucidate coping, including the conceptual approach (assuming a stable preference for coping strategies to deal with stress), the contextual approach (considering coping as a dynamic process), and the integrated approach. Within each theoretical framework, coping strategies (or styles) are classified according to two basic dimensions: the way a person behaves in a stressful situation (behavioural coping) on the one hand, and the emotions and cognitions a person attributes to the stressful situation (cognitive coping) on the other. In both dimensions, the most commonly used strategies are active (focus oriented or approaching) as opposed to passive (avoidant), these having supposedly favourable (adaptive) and unfavourable (maladaptive) effects, respectively. In particular, more avoidant behavioural coping has been related to worse functional outcome. ${ }^{3}$ The dimensions of coping considered and the strategies of interest can vary, depending on the stressors (generic or specific) and the outcome in question. ${ }^{4}$

In a study on labour force participation in patients with ankylosing spondylitis (AS), we showed that "coping with pain by decreasing activities" and "coping with limitations by pacing" were strong predictors of withdrawal from the labour force. ${ }^{5}$ Coping in this study was assessed by the "coping with rheumatic stressors" questionnaire (CORS), an arthritis specific coping questionnaire which measures eight coping strategies directed at the most important chronic stressors of inflammatory rheumatic disease: pain, limitations, and dependency. ${ }^{6}$ The two coping strategies identified as predictive of withdrawal from work are characterised as avoidant behavioural coping, as opposed to the six other strategies of CORS which reflect cognitive coping strategies.

Although that study was the first to demonstrate the importance of coping on functional outcome in AS, the relation has been recognised in rheumatoid arthritis. In 109 patients with rheumatoid arthritis it was shown that "decreasing activities to cope with pain" (CORS) was negatively associated with dexterity, while "pacing to cope with limitations" was positively associated with dexterity. In addition, "decreasing activities to cope with pain" was related to a decrease in dexterity after one year. ${ }^{7}$ In a cross sectional study among 720 patients with rheumatoid arthritis, avoidant coping (CORS) was associated with work disability. ${ }^{8}$ In 71 patients with rheumatoid arthritis, more frequent reassuring thoughts and less active (behavioural) coping (the Utrecht coping list) predicted worse result on the health assessment questionnaire (HAQ) at two years. ${ }^{9}$ These studies showed that the contribution of coping to functional outcome was independent of sociodemographics and current disease status. None of these studies addressed the issue of changes in coping strategies over time. ${ }^{6-9}$ However, this is important when interpreting cross sectional studies and when considering treatments aimed at changing coping strategies. As the characteristics of avoidant coping in patients with AS have not yet been examined, we report

\footnotetext{
Abbreviations: AS, ankylosing spondylitis; BASDAl, Bath ankylosing spondylitis disease activity index; BASFI, Bath ankylosing spondylitis functional index; CORS, "Coping with rheumatic stressors" questionnaire; $\mathrm{HAQ}$, health assessment questionnaire; ICC, intraclass correlation coefficient; OASIS, outcome assessment in ankylosing spondylitis international study; VAS, visual analogue scale
} 
here the extent to which two avoidant coping strategies in patients with AS-decreasing activities to cope with pain and pacing to cope with limitations-are influenced by the level of pain and physical limitations. In addition we determined whether these two coping strategies remained stable over a limited period of four years.

\section{METHODS \\ Patients}

In September 1997 a cross sectional sample of 658 patient with AS aged between 16 and 60 years, selected from the Dutch standard diagnosis register of rheumatic diseases (SDR), completed a postal questionnaire on sociodemographics, work status, and disease characteristics including coping. Ninety of these patients were part of a longitudinal study on outcome assessment in AS (OASIS), starting in 1997. Extensive health status assessments of these patients were carried out every six months during the first two years and yearly afterwards. At the fourth year evaluation, the questionnaire on coping was administered for a second time. Details of the patient selection procedures have been published previously ${ }^{1011}$ and can also be found in appendix 1 of the online version of this paper (available at http:// www.annrheumdis.com/supplemental).

\section{Questionnaires}

In the cross sectional survey, sociodemographic factors comprised sex, age, education, and work status. For further analyses educational level was dichotomised into more than 12 years of formal education and 12 years or less. In the cross sectional as well as in the follow up questionnaire, disease related variables included age at diagnosis, function measured by BASFI (Bath ankylosing spondylitis functional index), and pain measured by BASDAI (Bath ankylosing spondylitis disease activity index). The BASFI comprises 10 questions, each to be rated on a visual analogue scale (VAS; $0-10)$, on perceived limitations in daily activities. ${ }^{12}$ The final score ranges from 0 to 10 , and higher values indicate worse physical function. The BASDAI comprises six questions, each to be rated on a VAS $(0-10)$. Five items relate to pain and stiffness in different parts of the body caused by AS, and the remaining item relates to fatigue. ${ }^{13}$ The total score ranges from 0 to 10 , and higher values indicate greater disease activity.

Coping was assessed by the CORS, an arthritis specific and patient derived coping questionnaire which measures eight coping strategies directed at the most important chronic stressors of inflammatory rheumatic disease-pain, limitations, and dependency. Three scales refer to pain: comforting cognitions (nine items), decreasing activities (eight items), and diverting attention (eight items). Three scales measure strategies of coping with limitations: optimism (five items), pacing-that is, adapting one's level of activity (10 items), and creative solution seeking (eight items). Two scales refer to dependency: making effort to accept one's level of dependence (six items), and showing consideration (seven items). For each item, patients report how often they made use of that particular coping strategy $(\mathrm{l}=$ seldom or never, 2 = sometimes, $3=$ often, $4=$ very often), and each scale is calculated by summing the scores of the individual items. Higher scores indicate more frequent use of that specific coping style. The two scales, "decreasing activities to cope with pain" and "pacing to cope with limitations", are classified as the behavioural coping strategies, while all other six coping strategies are considered to be cognitive. The total score for the scale "decreasing activities to cope with pain" ranges from 8 to 32 , and for the scale "pacing to cope with limitations, from 10 to 40 . Higher values on the scale indicate more frequent use of this coping strategy. The CORS has documented reliability (Cronbach's $\alpha, 0.79$ to 0.88 ; Pearson's test-retest coefficient, 0.88 and 0.91 for "decreasing activities to cope with pain" and for "pacing to cope with limitations", respectively), and has been shown to have moderate to low correlation with the sociodemographic variables age, sex, education, and disease duration. ${ }^{7}$ A summary of the domains and scales with sample questions is available in appendix 2 of the online version of this article (http://www.annrheumdis. com/supplemental).

\section{Statistical analyses}

Characteristics of the patients were analysed using descriptive statistics. Linear regression was used to analyse the univariate relation $(n=658)$ between each of the two behavioural coping strategies and the following independent variables: sex, age, disease duration, educational level, physical function, and pain. Variables significantly associated with coping $(p<0.05)$ were included in a multiple linear regression model applying a backward elimination procedure to evaluate the independent contribution of the variables. For all independent variables we give the unstandardised coefficient (B), the standardised coefficient $(\beta)$, which allows comparison of the relative importance of the variables, and the adjusted correlation $\left(R^{2}\right)$, which allows assessment of the contribution of the variable to the explanation of the variability in coping. Agreement between scores of coping at the first assessment $(\mathrm{n}=70)$ and four years later was assessed by intraclass correlation coefficients (ICC). Bland and Altman plots were computed to visualise the difference between coping at the first and second assessment in individual patients. ${ }^{14}$ Correlations between change in coping over time and change in physical function (BASFI) or pain (BASDAI) over the same period were analysed by Pearson's correlation coefficient. Changes in score were calculated as the score at year 4 minus the score at the first assessment. Data were analysed by SPSS version 9.0 (SPSS Inc, Chicago, Illinois, USA).

\section{RESULTS \\ Patients}

In the initial cross sectional group $(n=658), 70 \%$ were male, their mean (SD) age was 43.5 (9.4) years, their mean disease duration was 12.3 (8.0) years, $80 \%$ had less than 12 years of formal education, and $20 \%$ had withdrawn from paid work because of disability. The 70 patients who completed the questionnaire again after four years did not differ in sociodemographic and disease characteristics from the patients in the national group except for a somewhat shorter disease duration $(10.5(7.6)$ years; $p=0.2)$. A detailed comparison between the groups is given in appendix 3 of the online version of this article (http://www.annrheumdis. com/supplemental).

\section{Avoidant behavioural coping and health status at the first assessment}

In patients from the initial cross sectional group the mean (SD) score for the scale "coping with pain by decreasing activities" was 18.4 (4.3) (interquartile range 15 to 22) and the proportion of patients using this coping strategy often or very often was $42 \%$. The mean score for the scale "coping with limitations by pacing" was 26.4 (6.0) (interquartile range 21 to 30 ) and the proportion of patients using this coping strategy often or very often was $62 \%$. As can be seen in table 1, worse physical function (BASFI) and more pain (BASDAI) were more strongly related to avoidant behavioural coping strategies than sociodemographic characteristics in univariate analysis. The multivariate analyses (table 2) showed that female sex ( $\mathrm{B}=1.28$ (95\% confidence interval (CI), 0.56 to 2.01$) ; \mathrm{p}=0.001$ ) and worse physical 
function $(\mathrm{B}=0.81(0.61$ to 0.99$) ; \mathrm{p}<0.001)$ were associated with the likelihood of decreasing activity to cope with pain (adjusted $\left.R^{2}=0.24\right)$, while female sex $(\mathrm{B}=1.21 \quad(0.25$ to $2.17) ; \mathrm{p}=0.01)$, older age $(\mathrm{B}=0.12 \quad(0.07$ to 0.17$)$; $\mathrm{p}<0.0001)$, and worse physical function $(\mathrm{B}=0.99$ (0.81 to $1.18) ; \mathrm{p}<0.0001$ ) were associated with likelihood of pacing to cope with limitations (adjusted $R^{2}=0.22$ ). In the multivariate analyses, the BASDAI made no independent contribution to coping, probably because of the strong correlation between BASDAI and BASFI $\left(R^{2}=0.70\right.$; $\mathrm{p}=0.01$ ), as can be seen online in appendix 4 (http:// www.annrheumdis.com/supplemental).

\section{Avoidant behavioural coping and change over time}

In the overall group, disease duration showed no association with coping at the first assessment in the multivariate analyses. In the 70 patients taking part in the longitudinal study, the mean (SD) score for decreasing activities to cope with pain at the first assessment was 18.4 (4.4) and at year 4, 19.2 (4.0). Pacing to cope with limitations at the first assessment was 25.9 (6.4) and at year 4, 27.9 (5.4). The mean (SD) difference in score for decreasing activities to cope with pain was 0.77 (4.6) (range -9 to 13), and for pacing to cope with limitations, 0.76 (5.6) (range -8 to 20).

Although the mean group values suggested small differences in coping over time, the ICCs suggested only moderate agreement. The ICC of the coping strategy "decreasing activities to cope with pain" at the first assessment and after four years was 0.56 ( 0.34 to 0.74$)$. The ICC of the coping strategy "pacing to cope with limitations" at baseline and four years later was 0.70 (0.53 to 0.82). The Bland and Altman plots can be seen in fig $1 \mathrm{~A}$ and $1 \mathrm{~B}$. Each dot represents one patient, with the mean coping of the two assessments on the $\mathrm{X}$ axis and difference in coping between the year 4 assessment and the first assessment on the $\mathrm{Y}$ axis. If there were no individual change in coping between the two assessments, all dots would be on the zero line of the $\mathrm{Y}$ axis (difference between coping at year 4 and at the first assessment). The plots in fig 1 suggest a difference in coping over time, with changes in coping strategies in both directions-some patients using the strategy more often and others less often. The change in coping over time could not be explained by the change in physical function or pain, as can be seen from the very low Pearson correlation coefficients of the change values of coping and health status (decreasing activities and BASFI, $0.03(\mathrm{p}=0.8)$; decreasing activities and BASDAI, $0.09(\mathrm{p}=0.4)$; pacing and BASFI, 0.07 $(p=0.6)$; pacing and BASDAI, $0.09(p=0.5)$. The absence of a relation between change in coping over time and change in health status (BASFI or BASDAI) could not be explained by ceiling or bottom effects in the coping strategy. Both coping strategies were normally distributed (skewness for coping with pain, -0.1 , and for coping with limitations, -0.3$)$, and few patients had a minimum or a maximum score (two patients had a minimum score: one for coping with pain, the other for coping with limitations; one patient had a maximum score, for coping with limitations).

\section{DISCUSSION}

In the framework of understanding functional outcome in chronic rheumatic diseases as proposed by the International Classification of Function (ICF), it is recognised that not only disease related factors but also psychosocial factors such as illness perception, depression, coping, and social support play an additional role. In a retrospective study, we identified avoidant behavioural but not cognitive coping strategies to be associated with withdrawal from the labour force in patients with AS. ${ }^{5}$ However, it remained insufficiently clear to what extent avoidant coping with pain and limitations were

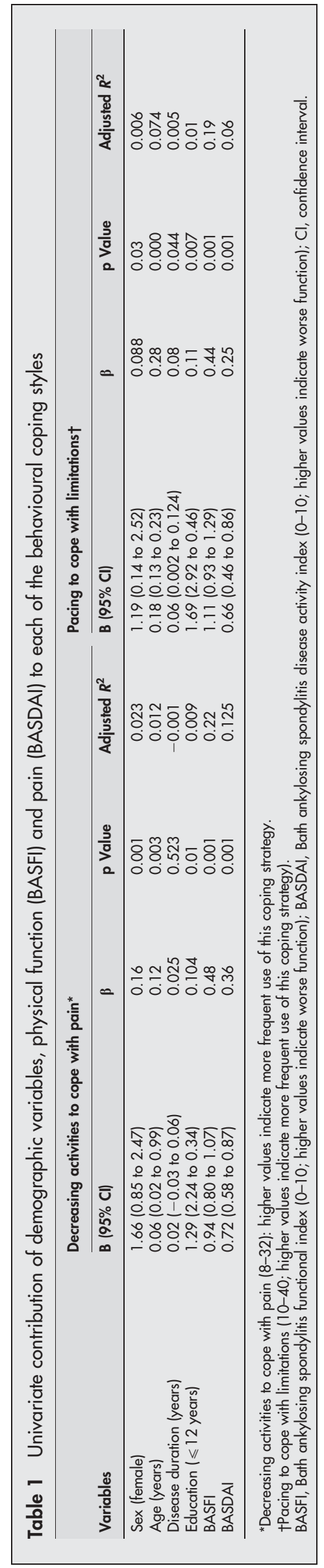



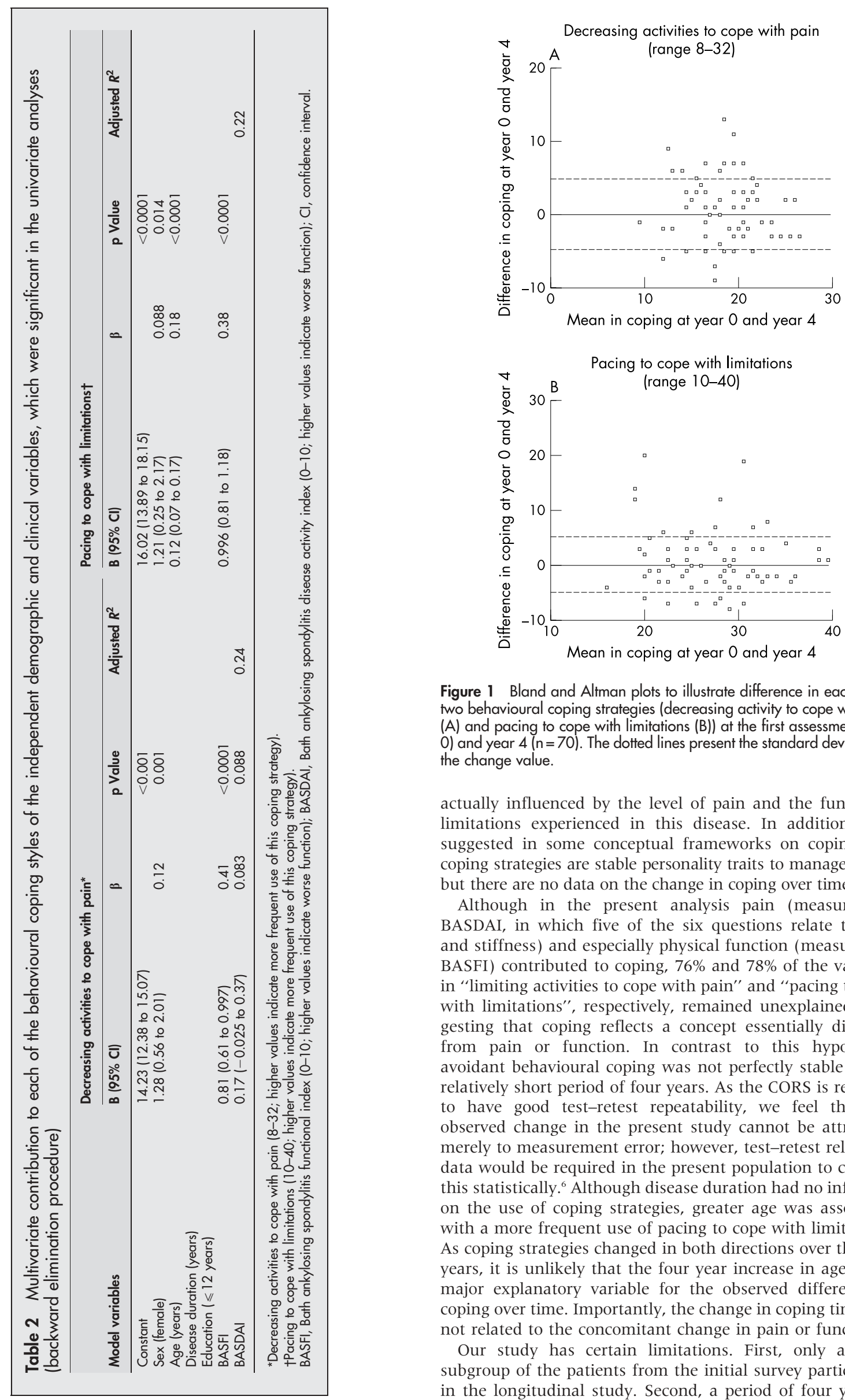

Figure 1 Bland and Altman plots to illustrate difference in each of the two behavioural coping strategies (decreasing activity to cope with pain (A) and pacing to cope with limitations (B)) at the first assessment (year $0)$ and year $4(n=70)$. The dotted lines present the standard deviation of the change value.

actually influenced by the level of pain and the functional limitations experienced in this disease. In addition, it is suggested in some conceptual frameworks on coping that coping strategies are stable personality traits to manage stress, but there are no data on the change in coping over time.

Although in the present analysis pain (measured by BASDAI, in which five of the six questions relate to pain and stiffness) and especially physical function (measured by BASFI) contributed to coping, $76 \%$ and $78 \%$ of the variance in "limiting activities to cope with pain" and "pacing to cope with limitations", respectively, remained unexplained, suggesting that coping reflects a concept essentially different from pain or function. In contrast to this hypothesis, avoidant behavioural coping was not perfectly stable over a relatively short period of four years. As the CORS is reported to have good test-retest repeatability, we feel that the observed change in the present study cannot be attributed merely to measurement error; however, test-retest reliability data would be required in the present population to confirm this statistically. ${ }^{6}$ Although disease duration had no influence on the use of coping strategies, greater age was associated with a more frequent use of pacing to cope with limitations. As coping strategies changed in both directions over the four years, it is unlikely that the four year increase in age was a major explanatory variable for the observed difference in coping over time. Importantly, the change in coping time was not related to the concomitant change in pain or function.

Our study has certain limitations. First, only a small subgroup of the patients from the initial survey participated in the longitudinal study. Second, a period of four years is 
short when evaluating a change in a personality trait such as coping. Third, the study did not allow estimating the smallest detectable difference, and no data are available on the clinical relevant difference in coping strategy; this would be useful for assessing the number of patients with a relevant change in coping strategy. Fourth, to study whether coping strategies are influenced by the presence and course of a disease, it is essential to assess coping from diagnosis onwards, and preferably even from before diagnosis. Also, for a comprehensive understanding of variables influencing coping, not only should disease related and sociodemographic variables be measured, but also psychological characteristics such as psychopathology, illness perception, and environmental barriers or facilitators. Finally, despite the fact that the CORS is a disease specific coping questionnaire, it would be valuable to compare the coping strategies and evolution of coping strategies over time with a control group. Also, normal individuals have a behavioural coping strategy when confronted with pain or limitations. The independent influence of a disease can therefore only be studied when compared with a control group from the general population.

If avoidant coping can vary over time, it might be possible to influence coping towards strategies that help to improve the functional outcome. In published reports on AS, we found only one randomised controlled trial in 168 patients with chronic rheumatic diseases including AS, aimed at improving "action directed coping" in patients with loneliness, lack of social support, or an impact of their disease on social behaviour. Patients were assigned to a specific coping intervention group (10 weekly sessions), a mutual support control group ( 10 weekly meetings), or a waiting list control group. ${ }^{15}$ Although the coping intervention group showed significantly more improvement in health status (on the sickness impact profile)—especially in the mobility domainat a three month evaluation, there was no significant difference between the mutual support control group and the waiting list controls. In addition, the effects were lost at a nine month follow up. Further intervention studies are needed with attention to patient selection, type and timing of the coping intervention, and the instruments to assess outcome.

Some hypotheses have been proposed in the literature to explain the adverse effect of avoidant behavioural coping on functional outcome. Avoidance, such as decreasing activities, and pacing, through expectancy of increased pain, can become maladaptive when used continuously. Avoidance behaviour may hence induce harmful long term effects on physical functioning by limiting joint movement and inducing muscle weakness. ${ }^{16-18}$ In this regard, the beneficial effects of exercise therapy in AS on functional outcome might well act through maintaining better behavioural coping by decreasing pain avoidance behaviour, rather than by influencing the disease process and causing a decrease in structural damage.

\section{Conclusions}

There is evidence that behavioural coping strategies are not completely stable over time, but this change seems independent of disease duration and disease related change in physical function and pain. As behavioural coping is associated with functional outcome, it would be of value to gain improved insight into the variables that influence coping and which might be modifiable.

\section{ACKNOWLEDGEMENTS}

We gratefully thank all rheumatologists and their co-workers of the following rheumatology centres for helping to recruit patients of the national cross sectional study: Sint-Antoniusziekenhuis Nieuwegein, Rode Kruisziekenhuis Den Haag, Bosch Medicentrum Den Bosch, Sint Laurentiusziekenhuis Roermond, Sint-Jansgasthuis Weert, Groene Hartziekenhuis Gouda, Albert Schweitzerziekenhuis
Dordrecht, Kennemer Gasthuis locatie Deo Haarlem, Lievensberg Ziekenhuis Bergen op Zoom, Tweesteden Ziekenhuis Tilburg, Rijnstate Ziekenhuis Arnhem, Sint-Maartenskliniek Nijmegen, Medisch Spectrum Twente Enschede, and Academisch Ziekenhuis Maastricht. For assessing the patients of the OASIS cohort at the year 4 evaluation, we gratefully acknowledge Liesbeth Heuft, rheumatologist at the University Hospital Maastricht.

\section{Authors' affiliations}

A Boonen, Department of Internal Medicine, Division of Rheumatology, University Hospital Maastricht, Netherlands

D van der Heijde, Department of Internal Medicine, Division of Rheumatology, University Hospital Maastricht and Caphri Research Institute, Netherlands

R Landewé, Department of Internal Medicine, Division of Rheumatology, University Hospital Maastricht and Atrium Medical Centre, Heerlen, Netherlands

A Chorus, Division of Public Health, TNO Prevention and Health, Leiden, Netherlands

W van Lankveld, Department of Rheumatology, Sint-Maarten Hospital, Niimegen, Netherlands

H Miedema, Netherlands Expert Centre for Work Related

Musculoskeletal Disorders, University Hospital Dijkzigt and Erasmus University Rotterdam, Netherlands

H van der Tempel, Department of Rheumatology, Maasland Ziekenhuis Sittard, Netherlands

S van der Linden, Department of Internal Medicine, Division of Rheumatology and Caphri Research Institute, University Hospital Maastricht, Netherlands

\section{REFERENCES}

1 Lazarus RS. Psychological stress and coping in adaptation and illness. Int J Psychiatry Med 1974;5:321-33.

2 Lazarus RS. Coping theory and research: past, present, and future. Psychosom Med 1993;55:234-47.

3 Zeidner M, Endler N. Handbook of coping. Theory, research and applications. New York: John Wiley and Sons, 1996.

4 Parker J, Endler N. Coping with coping assessment: a critical review. Eur J Personality 1992;6:321-44.

5 Boonen A, Chorus A, Miedema H, van der Heijde D, Landewe R, Schouten H, et al. Withdrawal from labour force due to work disability in patients with ankylosing spondylitis. Ann Rheum Dis 2001;60:1033-9.

6 van Lankveld W, Naring G, van der Staak C, van 't Pad Bosh P, van de Putte L. De ontwikkeling van de CORS. Coping met Reuma Stressoren. Gedrag en Gezondheid 1993;21:40-8 [In Dutch.].

7 van Lankveld W, Naring G, van 't Pad Bosch P, van de Putte L. Behavioral coping and physical functioning: the effect of adjusting the level of activity on observed dexterity. J Rheumatol 1999;26:1058-64.

8 Chorus AM, Miedema HS, Wevers CW, van der Linden S. Work factors and behavioural coping in relation to withdrawal from the labour force in patients with rheumatoid arthritis. Ann Rheum Dis 2001;60:1025-32.

9 Scharloo M, Kaptein AA, Weinman JA, Hazes JM, Breedveld FC, Rooijmans HG. Predicting functional status in patients with rheumatoid arthritis. J Rheumatol 1999;26:1686-93.

10 Boonen A, Chorus A, Miedema H, van der Heijde D, van der Tempel H, van der Linden S. Employment, work disability, and work days lost in patients with ankylosing spondylitis: a cross sectional study of Dutch patients. Ann Rheum Dis 2001;60:353-8.

11 Boonen A, van der Heijde D, Landewé R, Spoorenberg A, Schouten H, Rutten van Molken $M$, et al. Work status and productivity costs due to ankylosing spondylitis: comparison of three European countries. Ann Rheum Dis 2002;61:429-37.

12 Calin A, Garrett S, Whitelock H, Kennedy LG, O'Hea J, Mallorie P, et al. A new approach to defining functional ability in ankylosing spondylitis: the development of the Bath Ankylosing Spondylitis Functional Index. J Rheumatol 1994;21:2281-5.

13 Garrett S, Jenkinson T, Kennedy LG, Whitelock H, Gaisford P, Calin A. A new approach to defining disease status in ankylosing spondylitis: the Bath Ankylosing Spondylitis Disease Activity Index. J Rheumatol 1994;21:2286-91.

14 Bland JM, Altman DG. Measuring agreement in method comparison studies. Stat Methods Med Res 1999;8:135-60.

15 Savelkoul M, de Witte LP, Candel MJ, van der Tempel H, van den Borne B. Effects of a coping intervention on patients with rheumatic diseases: results of a randomized controlled trial. Arthritis Rheum 2001;45:69-76.

16 Vlaeyen JW, Kole-Snijders AM, Boeren RG, van Eek H. Fear of movement/ (re)injury in chronic low back pain and its relation to behavioral performance. Pain 1995;62:363-72.

17 Dekker J, Boot B, van der Woude LH, Bijlsma JW. Pain and disability in osteoarthritis: a review of biobehavioral mechanisms. J Behav Med 1992;15:189-214.

18 Kottke FJ. The effects of limitation of activity upon the human body. JAMA $1966 ; 196: 825-30$. 